\title{
A prospective investigation of dietary prebiotic intake and colorectal cancer risk in the EPIC-Oxford cohort
}

\author{
Carlota Castro-Espin ${ }^{1,2}$, Brittany Graham ${ }^{2}$, Paul N. Appleby ${ }^{2}$, Antonio Agudo $^{1}$, \\ Timothy J. Key ${ }^{2}$ and Aurora Perez-Cornago ${ }^{2}$ \\ ${ }^{1}$ Unit of Nutrition and Cancer, Cancer Epidemiology Research Program, Catalan Institute of Oncology-IDIBELL. \\ L'Hospitalet de Llobregat, Barcelona, Spain and \\ ${ }^{2}$ Cancer Epidemiology Unit, Nuffield Department of Population Health, University of Oxford, Oxford, United Kingdom
}

\section{Abstract}

Introduction: Prebiotics are a subtype of dietary fibre selectively fermented by beneficial bacterial in the colon. Preclinical evidence has suggested that prebiotics may be associated with a decreased risk of colorectal cancer. However, the association between dietary intake of prebiotics and colorectal cancer risk has not been investigated prospectively. This study aims to prospectively investigate the association between total prebiotic intake and colorectal cancer risk. Further characterisation of the association by prebiotic sub-type (fructans and galacto-oligosaccharides (GOSs)) and colorectal cancer sub-site (colon cancer and rectal cancer) were secondary objectives.

Material and methods: A total of 53,700 men and women living in England and Scotland who were enrolled in the European Prospective Investigation into Cancer and Nutrition (EPIC)-Oxford study, were included in the analysis and followed up for incident colorectal cancers. Validated semi-quantitative food frequency questionnaires administered at baseline were used to calculate daily fructan, GOS and total prebiotic intake. We used multivariable Cox proportional hazards models to assess associations between prebiotic intake and risk of colorectal cancer.

Results: A total of 574 incident cases of colorectal cancer were identified during a mean of 16.1 years of follow-up. Total prebiotic, fructan and GOS intake were not significantly associated with colorectal cancer risk. The hazard ratios for those in the highest fourths of total prebiotic, fructan and GOS intake compared to those in the lowest fourths were 0.87 (95\% confidence intervals (CI) $0.66-1.14$; $\mathrm{P}$ for trend $=0.3), 0.91(95 \%$ CI $0.70-1.18 ; \mathrm{P}$ for trend $=0.4)$, and $0.87(95 \%$ CI $0.66-1.15$; $\mathrm{P}$ for trend $=0.4)$ respectively. The associations remained nonsignificant when colorectal cancer sub-sites were investigated separately.

Discussion: The results from this observational study do not support an association between prebiotic intake and colorectal cancer risk. Given the biological plausibility of a role for prebiotics in reducing colorectal cancer risk and since the non-significant association between prebiotic intake and colorectal cancer risk observed in the current study may be due to the small number of cases and the healthy profile of the cohort, further epidemiological research is needed to characterise the association between dietary prebiotic intake and colorectal cancer incidence.

\section{Conflict of Interest}

There is no conflict of interest 